No. 580

December 2017

Numerical Benchmarking for 3D Multiphase

Flow: New Results for a Rising Bubble

S. Turek, O. Mierka, K. Bäumler

ISSN: 2190-1767 


\title{
Numerical Benchmarking for 3D Multiphase Flow: New Results for a Rising Bubble
}

\author{
Stefan Turek ${ }^{1}$, Otto Mierka ${ }^{1}$, and Kathrin Bäumler ${ }^{2}$ \\ 1 Institute for Applied Mathematics (LS3), TU Dortmund University, Germany, \\ stefan.turek@math.tu-dortmund.de \\ 2 Institute for Applied Mathematics (LS3), TU Dortmund University, Germany, \\ otto.mierka@math.tu-dortmund.de \\ 3 Department of Radiology, Stanford University, USA, baeumler@stanford.edu
}

\begin{abstract}
Based on the benchmark results in [1] for a $2 \mathrm{D}$ rising bubble, we present the extension towards $3 \mathrm{D}$ providing test cases with corresponding reference results, following the suggestions in [2]. Additionally, we include also an axisymmetric configuration which allows $2.5 \mathrm{D}$ simulations and which provides further possibilities for validation and evaluation of numerical multiphase flow components and software tools in $3 \mathrm{D}$.
\end{abstract}

\section{Introduction}

The aim of this short note is to present reference results for a 3D rising bubble benchmark which is based on the former $2 \mathrm{D}$ benchmark configuration in [1]. In a first step, we describe an 'easier' setting in an axisymmetric configuration which allows the rigorous comparison and validation of the used 3D methodology and software based on reference results obtained by a highly accurate 2.5D approach in [3]. Then, in the second step, we demonstrate the numerical convergence behaviour for the 3D configuration for (at least) 3 successively refined spatial meshes and time steps which demonstrate that the proposed reference values are (almost) grid independent. Finally, we compare the new results with the previously published results in [2] which are slightly improved in terms of accuracy so that a new validated set of reference data is available now which can be found and downloaded from www.featflow.de.

\subsection{Definition of the Benchmark configuration}

The presented benchmark considers isothermal, incompressible flow of two immiscible fluids. The conservation of momentum and mass is described by the Navier-Stokes equations

$$
\begin{aligned}
\rho(\mathbf{x})\left(\frac{\partial \mathbf{u}}{\partial t}+(\mathbf{u} \cdot \nabla) \mathbf{u}\right) & =-\nabla p+\nabla \cdot\left(\mu(\mathbf{x})\left(\nabla \mathbf{u}+(\nabla \mathbf{u})^{T}\right)\right)+\rho(\mathbf{x}) \mathbf{g} \\
\nabla \cdot \mathbf{u} & =0
\end{aligned}
$$


in a fixed space-time domain $\Omega \times[0, T]$, where $\Omega \subset \mathbb{R}^{3}$. Here, $\rho(\cdot)$ and $\mu(\cdot)$ denote the density and viscosity of the fluids, $\mathbf{u}$ the velocity, $p$ the pressure, and $\mathbf{g}$ the external gravitational force field. It is assumed that fluid 1 occupies the domain $\Omega_{1}$ and that it completely surrounds fluid 2 in $\Omega_{2}$ (see Figure 1), in particular $\Gamma:=\partial \Omega_{1} \cap \partial \Omega_{2}, \Omega=\Omega_{1} \cup \Gamma \cup \Omega_{2}$, and $\partial \Omega_{2} \cap \partial \Omega=\emptyset$.

Surface tension effects are taken into consideration through the following force balance at the interface $\Gamma$

$$
\left.[\mathbf{u}]\right|_{\Gamma}=0,\left.\quad\left[-p \mathbf{I}+\mu\left(\nabla \mathbf{u}+(\nabla \mathbf{u})^{T}\right)\right]\right|_{\Gamma} \cdot \hat{\mathbf{n}}=\sigma \kappa \hat{\mathbf{n}}
$$

where $\hat{\mathbf{n}}$ is the unit normal at the interface, $\sigma$ is the surface tension coefficient, $\kappa$ is the curvature of the interface.
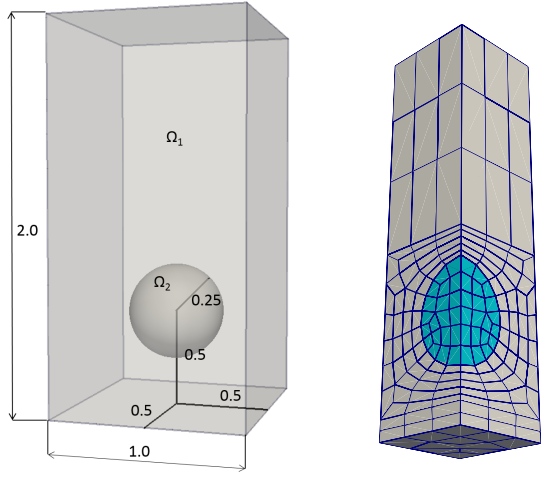

\begin{tabular}{llllll}
\hline$\rho_{1}$ & $\rho_{2}$ & $\mu_{1}$ & $\mu_{2}$ & $g$ & $\sigma$ \\
\hline 1000 & 100 & 10 & 1 & 0.98 & 24.5 \\
\hline & & & & \\
\hline L & $\#$ nel & & $\#$ nvt & & $\#$ dof $f_{\text {total }}$ \\
\hline 2 & 2,280 & 2,837 & 78,762 \\
3 & 18.240 & 20,377 & 596,958 \\
4 & 145.920 & 154,289 & $4,647,990$ \\
\hline
\end{tabular}

Fig. 1. Geometry with the corresponding Table 1. Dimensionless physical L1 mesh and initial condition of the 3D ris- parameters and geometrical statising bubble benchmark. tics of the 3D bubble benchmark.

The configuration of this benchmark problem is designed as an extension of its 2D predecessor benchmark introduced by Hysing et al. [1]. Besides the dimensional difference $(2 \mathrm{D} / 3 \mathrm{D})$ the only difference between the two benchmarks is the boundary condition imposed on the vertical sides of the domain being no-slip in the $3 \mathrm{D}$ case instead of the free-slip condition in the $2 \mathrm{D}$ case. This difference originates from the pioneering work of Adelsberger et al. [2], where such an adaptation has been chosen. The geometric description of the benchmark at initial condition is displayed at Fig. 1. All walls of the domain are characterized by no-slip boundary conditions.

The considered benchmark quantities are selected as for the original 2D benchmark, namely:

- Bubble Size - Size of the bubble in two different directions, namely in the rise direction $(z)$ expressed as $R_{z} / R_{0}$ (normalized w.r.t. initial bubble 
size $R_{0}$ ) and in a perpendicular-to-rise direction being aligned with one of the cartesian axis ( $x$ or $y$ ) expressed as $R_{x, y} / R_{0}$.

- Bubble Sphericity - The "degree of sphericity" in $\mathbb{R}^{3}$ be defined as

$$
\frac{A_{0}}{A}=\frac{4 \pi R_{0}^{2}}{A} \text {. }
$$

Here, $A_{0}$ denotes the area of a sphere with the initial bubble diameter $R_{0}$ which has a volume equal to that of the bubble with area $A$.

- Rise Velocity - The mean velocity with which the bubble is rising or moving and is defined as

$$
\mathbf{U}_{c}=\frac{\int_{\Omega_{2}} \mathbf{u} d x}{\int_{\Omega_{2}} 1 d x}
$$

where $\Omega_{2}$ denotes the region that the bubble occupies. The velocity component in the direction opposite to the gravity vector is then denoted as rise velocity $V_{c}$, for which the stationary limit is called terminal velocity.

\subsection{Description of the used numerical techniques}

The 3D results are based on a specific extension ('FeatFlower') of the FEM based open-source software package FeatFlow [4] which is a 3D multiphase CFD package (see also [5]) associated with the following key attributes:

- parallelized on the basis of domain decomposition technique

- equipped with geometrical (Newton)-multigrid solvers

- using higher order $Q_{2} / P_{1}$ iso-parametric elements

- using semi-implicit surface tension treatment on the basis of the LaplaceBeltrami transformation

- using interface-aligned, moving meshes in the framework of PDE based mesh deformation techniques together with the Arbitrary-LagrangianEulerian method

The main components of the $2.5 \mathrm{D}$ software by $\mathrm{K}$. Bäumler which is described in [3] can be shortly listed as follows:

- iso-parametric P2/P1 finite elements

- semi-implicit treatment of surface tension via Laplace-Beltrami transformation

- interface-aligned meshes which are moving in an ALE framework

- use of a reference frame fixing the center of mass of the bubble

- subspace projection method for implementing interface conditions

\section{Numerical simulation results}

First, the validation of the 3D code w.r.t. the 2.5D approach for the axisymmetric configuration will be presented, demonstrating the temporal and spatial convergence behaviour, before switching to the fully 3D benchmark test case. 


\subsection{Validation of the $3 \mathrm{D}$ results via $2.5 \mathrm{D}$ configuration}

The corresponding results in Figure 2 provide the results for different mesh levels and time steps and demonstrate that the 3D results are more or less independent of the chosen spatial refinement levels and that they agree, for a sufficiently small time step, very well with the results by the $2.5 \mathrm{D}$ approach. Based on these results and the corresponding numerical analysis, we claim that the applied 3D code is validated and is able to reproduce the 'reference results' which have been calculated via the special $2.5 \mathrm{D}$ code.

\subsection{D Benchmark results}

Having validated the 3D multiphase flow solver via the shown axisymmetric configuration, we perform the analogous simulations for the described full 3D benchmark case. Again, we provide in Table 2 the results for different mesh levels in space and time for selected benchmark quantities and mark the resulting reference results: Here, we use the LS3 results with the smallest time step size as reference values since the simulations show that the higher mesh level L4 leads to almost identical values as compared with level L3. Moreover, we provide in Figure 3 the corresponding temporal plots for the described benchmark quantities. Finally, we compare in Figure 4 the 'new' reference results with the previously published results in [2].

\section{Conclusions}

In this short paper, we have described the settings for a quantitative 3D Rising Bubble benchmark which is based on the previous studies in [1] and [2]. After validating the $3 \mathrm{D}$ code via an axisymmetric configuration (allowing to compare with corresponding highly accurate $2 \mathrm{D}$ simulations), we provide (new) reference benchmark quantities which all can be downloaded from www. featflow.de. A more detailed numerical analysis of the benchmark simulations as well as a more detailed description of the used methodology and codes will be part of a forthcoming paper.

\section{Acknowledgments}

The financial support of DFG (SPP 1740) is gratefully acknowledged (TU 102/53-1). The computations have been carried out on the LiDOng cluster at TU Dortmund University. We would like to thank the LiDOng cluster team for their help and support. 
Table 2. Convergence of the monitored quantities at $T=1.0$ and at final time $T=3.0$ w.r.t. temporal and spatial refinements. Explanation of the symbols is as follows: L - spatial refinement level, $\Delta t$ is the used time step, $V_{0}, V$ are the initial and actual volumes of the bubble, $z_{c}$ is the $z$ location of the bubble associated with its point of mass. The remaining parameters are introduced in Section 1.2.

\begin{tabular}{|c|c|c|c|c|c|c|c|}
\hline $\mathrm{L}$ & $\frac{\Delta t}{1 e-3}$ & $A_{0} / A$ & $\frac{V_{0}-V}{V_{0}} \%$ & $z_{c}$ & $V_{c}$ & $R_{z} / R_{0}$ & $R_{x, y} / R_{0}$ \\
\hline & & & & \multicolumn{4}{|l|}{$T=1.0$} \\
\hline 2 & $1 / 1$ & 0.97361 & 0.14353 & 0.27509 & 0.35653 & 0.78862 & 1.10488 \\
\hline 2 & $1 / 4$ & 0.97417 & 0.03115 & 0.27553 & 0.35694 & 0.78778 & 1.10560 \\
\hline 2 & $1 / 16$ & 0.97433 & 0.00274 & 0.27553 & 0.35707 & 0.78763 & 1.10555 \\
\hline 3 & $1 / 1$ & 0.97355 & 0.15011 & 0.27513 & 0.35652 & 0.78858 & 1.10580 \\
\hline 3 & $1 / 4$ & 0.97408 & 0.03768 & 0.27560 & 0.35695 & 0.78763 & 1.10593 \\
\hline 3 & $1 / 16$ & 0.97421 & 0.00940 & 0.27568 & 0.35707 & 0.78739 & 1.10596 \\
\hline 3 & $1 / 64$ & 0.97418 & 0.00140 & 0.27583 & 0.35709 & 0.78708 & 1.10608 \\
\hline 4 & $1 / 1$ & 0.97354 & 0.15005 & 0.27514 & 0.35650 & 0.78855 & 1.10576 \\
\hline \multirow[t]{2}{*}{4} & $1 / 4$ & 0.97406 & 0.03781 & 0.27561 & 0.35695 & 0.78757 & 1.10594 \\
\hline & & & & \multicolumn{4}{|l|}{$T=3.0$} \\
\hline 2 & $1 / 1$ & 0.95579 & 0.60085 & 0.97041 & 0.34779 & 0.73614 & 1.15893 \\
\hline 2 & $1 / 4$ & 0.95861 & 0.12664 & 0.97208 & 0.34855 & 0.73424 & 1.15676 \\
\hline 2 & $1 / 16$ & 0.95933 & 0.00573 & 0.97240 & 0.34875 & 0.73375 & 1.15660 \\
\hline 3 & $1 / 1$ & 0.95553 & 0.63544 & 0.97031 & 0.34775 & 0.73622 & 1.15919 \\
\hline 3 & $1 / 4$ & 0.95835 & 0.16248 & 0.97203 & 0.34851 & 0.73430 & 1.15806 \\
\hline 3 & $1 / 16$ & 0.95906 & 0.04294 & 0.97242 & 0.34871 & 0.73382 & 1.15778 \\
\hline 3 & $1 / 64$ & 0.95925 & 0.00952 & 0.97266 & 0.34876 & 0.73368 & 1.15776 \\
\hline 4 & $1 / 1$ & 0.95556 & 0.63182 & 0.97030 & 0.34775 & 0.73623 & 1.15932 \\
\hline 4 & $1 / 4$ & 0.95835 & 0.16107 & 0.97203 & 0.34851 & 0.73431 & 1.15820 \\
\hline
\end{tabular}

\section{References}

1. Hysing, S.; Turek, S.; Kuzmin, D.; Parolini, N.; Burman, E.; Ganesan, S.; TOBISKA, L., Quantitative benchmark computations of two-dimensional bubble dynamics. Int. J. Num. Meth. Fluids, 60, 11 (2009), 1259-1288.

2. J. Adelsberger, P. Esser, M. Griebel, S. Gross, M. Klitz, And A. RTTGERS, 3D incompressible two-phase flow benchmark computations for rising droplets, Proceedings of the 11th World Congress on Computational Mechanics (WCCM XI), Barcelona, 2014.

3. K. B̈̈UMLER, Simulation of single drops with variable interfacial tension, $\mathrm{PhD}$ Thesis, Friedrich-Alexander-Universität Erlangen-Nürnberg, 2014.

4. http://www.featflow.de

5. S. Turek, O. Mierka, S. Hysing, D. Kuzmin, Numerical study of a high order 3D FEM-Level Set approach for immiscible flow simulation, Computational Methods in Applied Sciences (S. Repin, T. Tilnonen, T. Tuovinen, eds.), Springer Verlag, 2013, pp. 65-91. 
S. Turek et al.
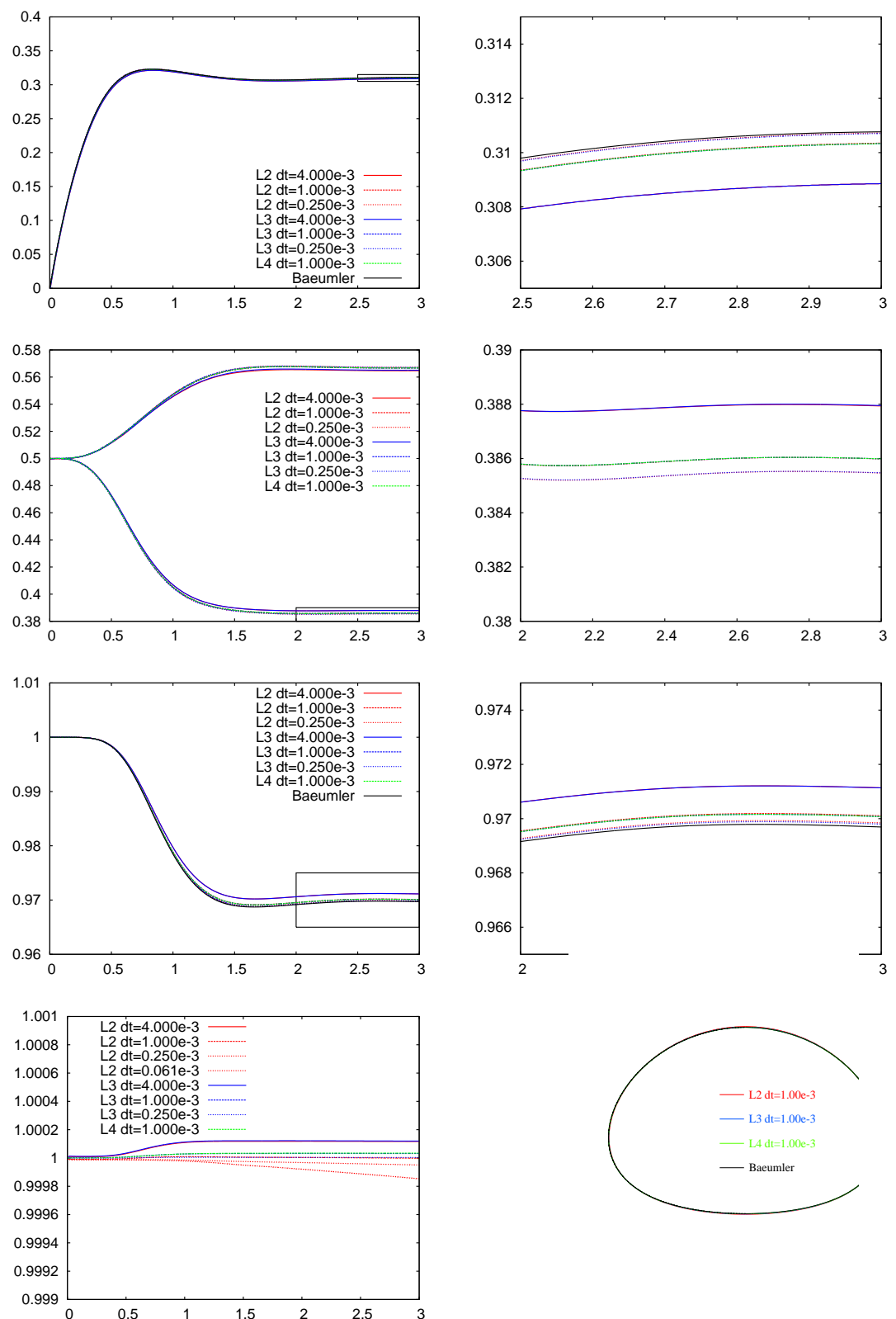

Fig. 2. From top to bottom: Evolution of bubble rise velocity $V_{c}$, bubble diameter $2 R_{z}, 2 R_{x, y}$, bubble sphericity $A_{0} / A$, relative bubble mass $V / V_{0}$ and bubble shape for the axisymmetric validation case. L2, L3, L4 represent the level of spatial resolutions and $d t$ stands for the size of the corresponding time step. 

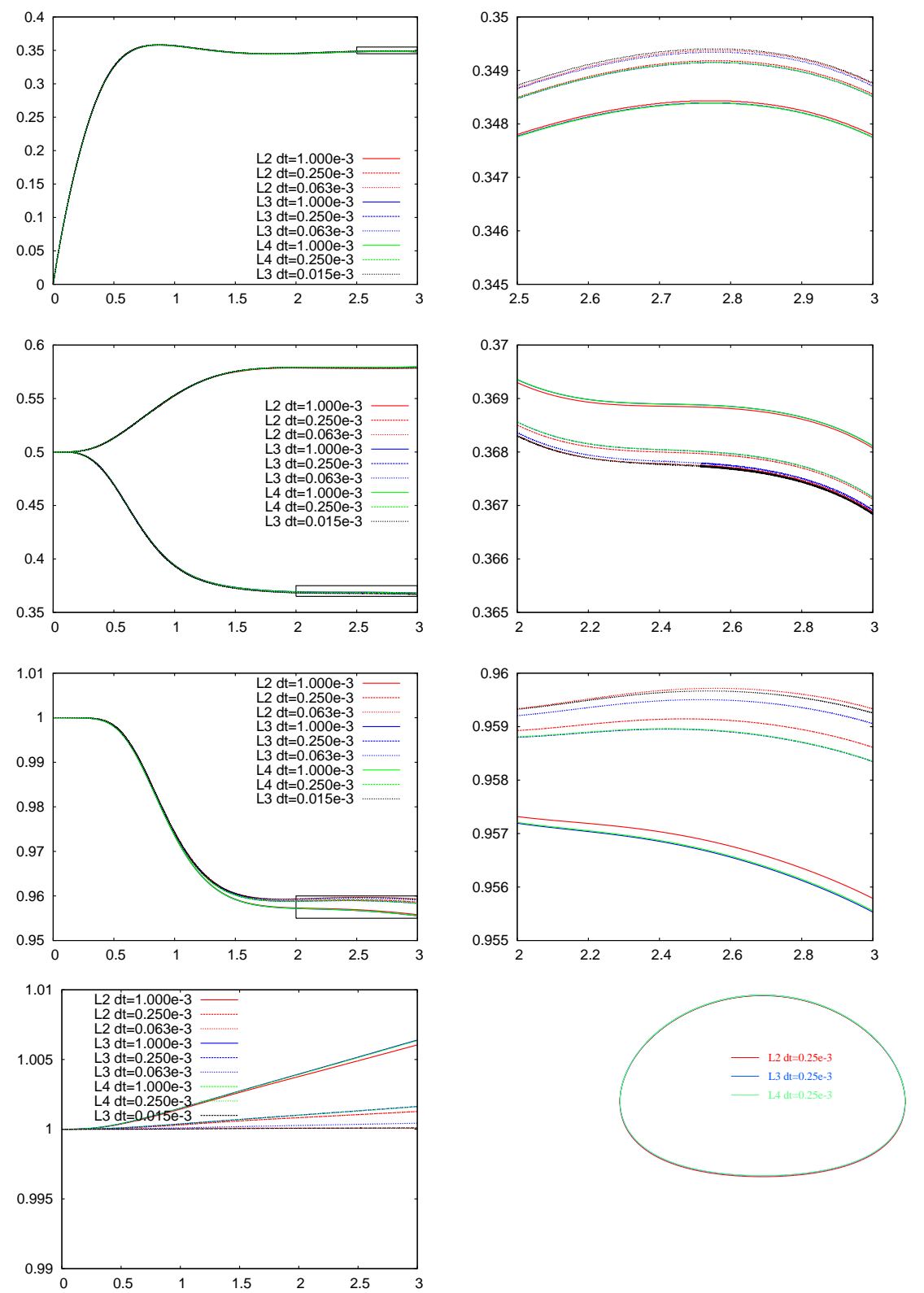

Fig. 3. From top to bottom: Evolution of bubble rise velocity $V_{c}$, bubble diameter $2 R_{z}, 2 R_{x, y}$, bubble sphericity $A_{0} / A$, relative bubble mass $V / V_{0}$ and bubble shape for the 3D benchmark problem. L2, L3, L4 represent the level of spatial resolutions and $d t$ stands for the size of the corresponding time step. 

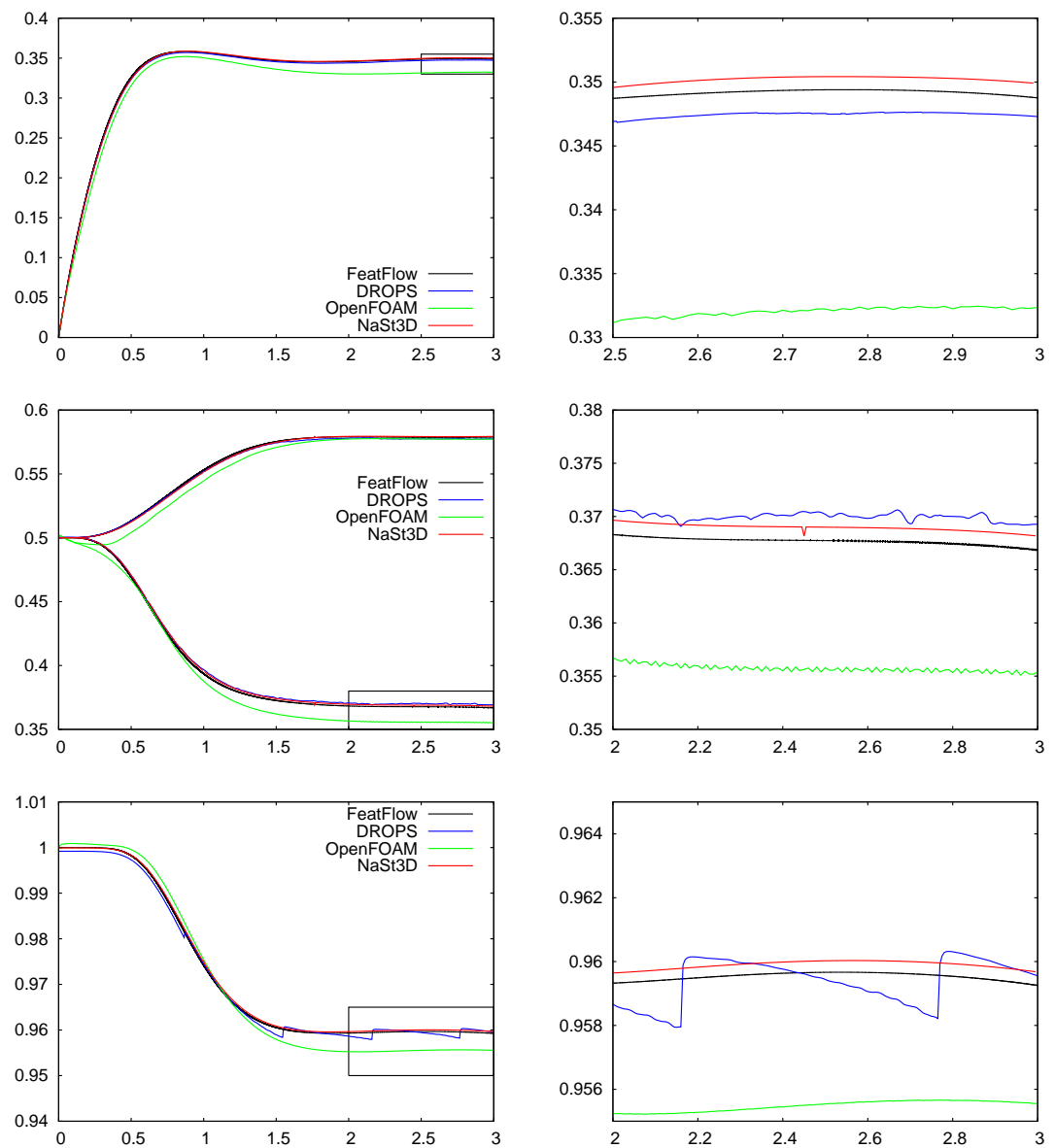

Fig. 4. From top to bottom: Evolution of bubble rise velocity $V_{c}$, bubble diameter $2 R_{z}, 2 R_{x, y}$ and bubble sphericity $A_{0} / A$ in comparison with the results published by Adelsberger et al. [2]. 